\title{
THE SUM OF TWO S-UNITS BEING A PERFECT POWER IN GLOBAL FUNCTION FIELDS
}

\author{
ISTVÁN GAÁL * AND MICHAEL POHST**
}

\begin{abstract}
Let $x_{1}$ and $x_{2}$ be integers divisible only by some fixed primes. Is it possible that $x_{1}+x_{2}$ is a perfect power? Special cases of the equation $x_{1}+x_{2}=y^{k}$ were formerly considered over $\mathbb{Z}$. In this paper we develop an algorithm to solve this equation over global algebraic function fields. Our method is illustrated by two explicit examples.
\end{abstract}

\section{INTRODUCTION}

Let $K$ be a number field and consider the equation

$$
x_{1}+x_{2}=1
$$

in units $x_{1}, x_{2}$ of $K$. This equation is called unit equation and plays an essential role in the resolution of several important types of diophantine equations, like Thue equations, norm form equations, index form equations etc, see [2].

B.M.M. de Weger [8] considered an analogous equation which can be treated as a generalization of unit equations. Let $S$ be a finite set of primes and denote by $H_{S}$ the set of those integers which are only divisible by primes from $S$. Consider the equation

$$
x_{1}+x_{2}=y^{2}
$$

in $x_{1}, x_{2} \in H_{S}$ and $y \in \mathbb{Z}$. The more general equation

$$
x_{1}+x_{2}=y^{k}
$$

in $x_{1}, x_{2} \in H_{S}, y \in \mathbb{Z}$ and unknown exponent $k$ appears in the book of Shorey and Tijdeman [7] (Chapter 9). As a preparatory step to the study of Catalan's equation they show that the prime divisors of the exponent $k$ can be bounded by an explicit constant.

1991 Mathematics Subject Classification. Primary 11Y50; Secondary 11D61.

Key words and phrases. S-units; global function fields.

Research supported in part by K67580 and K75566 from the Hungarian National Foundation for Scientific Research, by the MTA-DFG Project DFG/200 and by the TÁMOP 4.2.1./B09/1/KONV-2010-0007 project implemented through the New Hungary Development Plan co-financed by the European Social Fund, and the European Regional Development Fund . 
Recently, we extended the study of unit equations in two and more variables to the case of algebraic function fields over finite fields, see [3], [4]. This gives the basis to consider equations of type (1) over global function fields.

In our paper we develop an algorithm to solve equations of type (1) over function fields. We illustrate our method by two detailed examples. For the explicit calculations we used KANT [1].

\section{The FUNCTION FIELD}

Let $\mathbb{F}_{q}$ be a finite field with $q=p^{\ell}$ elements. We denote by $K$ the rational function field $\mathbb{F}_{q}(t)$. Our arguments are also valid if $K$ is a finite extension of $\mathbb{F}_{q}(t)$ having only one infinite valuation. Such extensions are e.g. Artin-Schreier extensions, see [6] (Section VI.6) and Example 2.

$O_{K}$ will denote $\mathbb{F}_{q}[t]$ if $K=\mathbb{F}_{q}(t)$ and the integral closure of $\mathbb{F}_{q}[t]$ in $K$ otherwise. We denote by $g$ the genus of $K$, which is zero if $K=\mathbb{F}_{q}(t)$.

The set of all (exponential) valuations of $K$ is denoted by $V$, the single infinite valuation by $v_{\infty}$. For a non-zero element $f \in K$ we denote by $v(f)$ the value of $f$ at $v$. For the normalized valuations $v_{N}(f)=v(f) \cdot \operatorname{deg} v$ the product formula

$$
\sum_{v \in V} v_{N}(f)=0, \quad \text { for all } f \in K \backslash\{0\}
$$

holds. The height of a non-zero element $f$ of $K$ is defined as

$$
H(f):=\sum_{v \in V} \max \left\{0, v_{N}(f)\right\}=-\sum_{v \in V} \min \left\{0, v_{N}(f)\right\} .
$$

\section{Formulating the statement}

Let $S$ be a finite set of valuations of $K$ including the infinite valuation. Denote by $H_{S}$ the set of those elements of $O_{K}$ which have zero values for all valuations outside $S$, that is

$$
H_{S}=\left\{\alpha \in K \mid v(\alpha)=0 \text { for all } v \in V \backslash S, v(\alpha) \geq 0 \text { for all } v \in S \backslash\left\{v_{\infty}\right\}\right\} .
$$

In case $K=\mathbb{F}_{q}(t)$ the set $S$ consists of finite valuations corresponding with some irreducible polynomials and the degree (infinite) valuation. The set $H_{S}$ is just the set of those polynomials in $\mathbb{F}_{q}[t]$ that are only divisible by these polynomials.

We consider the equation

$$
x_{1}+x_{2}=c \cdot y^{k} \quad \text { in } x_{1}, x_{2} \in H_{S}, 0 \neq c \in \mathbb{F}_{q}, y \in O_{K} \backslash \mathbb{F}_{q}, 2 \leq k \in \mathbb{Z} .
$$

Note that in characteristic $p$ an equation $x_{1}+x_{2}=y^{k}$ implies $x_{1}^{p}+x_{2}^{p}=y^{p k}$. Also, for $z \in H_{S}$, we have $z^{k} x_{1}+z^{k} x_{2}=(z y)^{k}$. Therefore, to avoid infinitely many solutions for trivial reasons we assume that

$$
k \geq 2, p \nmid k, v(y)=0 \text { for all } v \in S \backslash\left\{v_{\infty}\right\} .
$$


Remark. Instead of $p \nmid k$ it would also be possible to assume that $x_{1} / y^{k}$ is not a $p$-th power.

Our main result is the following theorem.

Theorem 1. Under the assumptions above equation (2) has (up to constant factors) only finitely many solutions that can be explicitely determined. Any solution satisfies

$$
\max \left(H\left(x_{1}\right), H\left(x_{2}\right)\right) \leq 2 \cdot\left(2 g-2+\sum_{v \in S} \operatorname{deg} v\right)
$$

In our proof we shall use the following Lemma on unit equations in two variables. This is valid in any algebraic function field $K$ over finite fields. Let $W$ be a finite set of valuation of $K$ including the infinite valuations. Note that a $W$-unit is an element of $K$ having zero values for all valuations outside $W$.

Lemma 2. Let $\gamma_{1}, \gamma_{2}$ be $W$-units satisfying

$$
\gamma_{1}+\gamma_{2}=1
$$

Then either $\gamma_{1}, \gamma_{2}$ are in $K^{p}$ or their heights are bounded:

$$
\max \left(H\left(\gamma_{1}\right), H\left(\gamma_{2}\right)\right) \leq 2 g-2+\sum_{v \in W} \operatorname{deg} v .
$$

For the proof of the Lemma see [3].

Proof of the theorem

Equation (2) implies

$$
\frac{x_{1}}{c y^{k}}+\frac{x_{2}}{c y^{k}}=1
$$

Let

$$
S(y)=\{v \mid v(y)>0, v \notin S\}
$$

and set $S_{1}=S \cup S(y)$. Then both terms on the left-hand side of (6) are $S_{1}$-units. By our assumptions in (2) and (3) $y$ is non-constant, $p \wedge k$ and in $x_{1}, x_{2}$ and $y$ only distinct finite valuations can attain positive values. Therefore, $x_{1} / y^{k}$, $x_{2} / y^{k}$ are not $p$-th powers. Hence, the Lemma implies that for $i=1,2$ we have

$$
H\left(\frac{x_{i}}{y^{k}}\right) \leq 2 g-2+\sum_{v \in S} \operatorname{deg} v+\sum_{v \in S(y)} \operatorname{deg} v
$$


Utilizing again that by (2) for infinite valuations $v$ the inequality $v(y)>0$ is tantamount to $v\left(y^{k} / x_{i}\right)>0$ and those values coincide for $i=1,2$ we get

$$
\begin{aligned}
& k \cdot \sum_{v \in S(y)} \operatorname{deg} v=k \cdot \sum_{v(y)>0, v \text { finite }} \operatorname{deg} v \\
& \leq k \cdot \sum_{v(y)>0, v \text { finite }} v(y) \operatorname{deg} v=\sum_{v(y)>0, v \text { finite }} v\left(y^{k}\right) \operatorname{deg} v \\
& =\sum_{v\left(y^{k} / x_{i}\right)>0, v \text { finite }} v\left(\frac{y^{k}}{x_{i}}\right) \operatorname{deg} v \leq \sum_{v\left(y^{k} / x_{i}\right)>0} v\left(\frac{y^{k}}{x_{i}}\right) \operatorname{deg} v \\
& =H\left(\frac{y^{k}}{x_{i}}\right)=H\left(\frac{x_{i}}{y^{k}}\right) \leq 2 g-2+\sum_{v \in S} \operatorname{deg} v+\sum_{v \in S(y)} \operatorname{deg} v,
\end{aligned}
$$

where the last inequality follows from (7). As a consequence we have

$$
(k-1) \sum_{v \in S(y)} \operatorname{deg} v \leq 2 g-2+\sum_{v \in S} \operatorname{deg} v .
$$

This inequality implies an upper bound for $k$ and $\sum_{v \in S(y)} \operatorname{deg} v$ which allows to determine the possible valuations in $S(y)$. Combining this with (7) we obtain

$$
H\left(\frac{x_{i}}{y^{k}}\right) \leq\left(1+\frac{1}{k-1}\right)\left(2 g-2+\sum_{v \in S} \operatorname{deg} v\right) .
$$

For our purposes we proceed as follows. In the first step we use our assumption that $K$ has only one infinite valuation. Then the conditions in (2) imply

$$
\begin{aligned}
& H\left(x_{i}\right)=\sum_{v\left(x_{i}\right)>0, v \text { finite }} v\left(x_{i}\right) \operatorname{deg} v=\sum_{v\left(x_{i} / y^{k}\right)>0, v \text { finite }} v\left(\frac{x_{i}}{y^{k}}\right) \operatorname{deg} v \\
& =\sum_{v\left(x_{i} / y^{k}\right)>0} v\left(\frac{x_{i}}{y^{k}}\right) \operatorname{deg} v=H\left(\frac{x_{i}}{y^{k}}\right) \\
& \leq\left(1+\frac{1}{k-1}\right)\left(2 g-2+\sum_{v \in S} \operatorname{deg} v\right) .
\end{aligned}
$$

In the last step we used (9). Inequality (10) implies the assertion (4) in the theorem. The proof is complete since in $K$ there exist only finitely many elements of bounded height which can be explicitely determined.

\section{The Algorithm to Determine the SOlutions}

As we know an upper bound for the heights of $x_{1}, x_{2} \in H_{S}$, it is very easy and fast to enumerate all possible $x_{1}$ and $x_{2}$. This is done just by taking the 
divisors corresponding to the finite valuations of $S$, composing power products of them with non negative exponents and with heights under the given bound. We check if the divisor is a principal divisor of $K$ generated by a single element. Listing all possible values of $x_{1}, x_{2}$ we can test if their sum is a perfect power satisfying the assumptions.

\section{EXAMPLES}

We illustrate our results by two detailed examples.

5.1. Example 1. Consider the polynomials $x_{1}, x_{2}$ over $\mathbb{F}_{5}$ that are only divisible by $t+1$ and $t^{2}+t+2$. For which $x_{1}$ and $x_{2}$ do we have

$$
x_{1}+x_{2}=c y^{k}
$$

a perfect power?

The rational function field $K=\mathbb{F}_{5}(t)$ has genus $0 . \quad S$ consists of the two irreducible polynomials $t+1$ and $t^{2}+t+2$ having degrees 1 and 2, respectively, and the infinite (degree) valuation of degree 1 . The theorem gives

$$
H\left(x_{i}\right) \leq 2(-2+4)=4 \text {. }
$$

Therefore

$$
x_{i}=c_{i}(t+1)^{r_{i}}\left(t^{2}+t+2\right)^{s_{i}}
$$

where $r_{i}, s_{i}$ are non negative and $r_{i}+2 s_{i} \leq 4$. Testing all possible $x_{1}+x_{2}$ we get the following solutions:

$$
\begin{aligned}
1+3\left(t^{2}+t+2\right) & =3(t+3)^{2} \\
1+3\left(t^{2}+t+2\right)(t+1) & =3(t+4)^{3} \\
(t+1)^{2}+\left(t^{2}+t+2\right) & =2(t+2)^{2} \\
(t+1)^{3}+2\left(t^{2}+t+2\right) & =t^{3} .
\end{aligned}
$$

All other solutions are obtained from those upon multiplication by a constant or by interchanging $x_{1}$ and $x_{2}$.

5.2. Example 2. Consider the function field $K$ generated by a root $\alpha$ of the polynomial

$$
f(y)=y^{3}-y-t
$$

over $\mathbb{F}_{3}(t)$. This is a so called Artin-Schreier extension, see [6]. $F$ has genus zero, in $F$ there is only one infinite valuation $v_{\infty}$ of degree 1 and there are three valuations $v_{1}, v_{2}, v_{3}$ of degree 2 extending the valuation of $\mathbb{F}_{3}[t]$ corresponding to the polynomial $t^{2}+1$. Let

$$
S=\left\{v_{\infty}, v_{1}, v_{2}, v_{3}\right\}
$$


Consider the equation

$x_{1}+x_{2}=c \cdot y^{k} \quad$ in $x_{1}, x_{2} \in H_{S}, 0 \neq c \in \mathbb{F}_{q}, y \in O_{K} \backslash \mathbb{F}_{q}, 2 \leq k \in \mathbb{Z}, 3 \not k$.

We have

$$
H\left(x_{i}\right) \leq 2(-2+7)=10 .
$$

Up to constant factors there are 56 elements in $H_{S}$ satisfying this condition. We tested all $x_{1}+x_{2}$ (with all possible constant factors) and obtained the solutions listed below. In the table we list $x_{1}, x_{2}, c, y$ ( $c$ a constant) satisfying

$$
x_{1}+x_{2}=c \cdot y^{2} .
$$

(Note that for some $x_{1}+x_{2}$ we also obtained cubes but these were excluded because of the assumption $p \nmid k$ ). For $x_{1}, x_{2}, y$ we give their coefficients $\left(u_{0}, u_{1}, u_{2}\right)$ in the basis $1, \alpha, \alpha^{2}$ of $K$. One of the lines correspond to the trivial solution

$$
\left(t^{2}+1\right)+2=t^{2}
$$

which was the initiative of this example. All further solutions are given by constant multiples of these or by interchanging $x_{1}$ and $x_{2}$.

$$
\begin{array}{cc}
x_{1}, x_{2} & c, y \\
(2,0,0),(2,1,1) & 1,(2,1,0) \\
(1,0,0),(1,1,2) & 2,(1,1,0) \\
(1,0,0),(2,2 t, 2) & 2,(0,0,1) \\
(1,0,0),(2,0,2) & 2,(0,1,0) \\
(1,0,0),(2 t+1,2 t+1,2) & 2,(2,1,2) \\
(1,0,0),(t+1,2 t+2,2) & 2,(1,1,1) \\
(1,0,0),\left(2 t^{2}+2,0,0\right) & 2,(t, 0,0) \\
(1,0,0),\left(2 t^{2}+2,2 t^{3}+2 t, 2 t^{2}+2\right) & 2,(0,2, t) \\
(1,0,0),\left(2 t^{3}+t^{2}+2 t+1,2 t^{3}+t^{2}+2 t+1,2 t^{2}+2\right) & 2,(t+2,2 t+2, t) \\
(1,0,0),\left(t^{3}+t^{2}+t+1,2 t^{3}+2 t^{2}+2 t+2,2 t^{2}+2\right) & 2,(t+1, t+2, t) \\
(1, t, 1),(1, t, 0) & 2,(2,0,1) \\
(1, t, 1),(2,2 t, 0) & 1,(0,1,0) \\
(t+1, t, 0),(t+2, t+2,1) & 2,(0,2,1) \\
(t+1, t, 0),(2 t+1,2 t+1,2) & 2,(1,1,0) \\
(t+1, t, 0),(1, t, 0) & 2,(2,1,1) \\
(2 t+1, t, 0),(t+1, t, 0) & 2,(1,0,1) \\
(2 t+1, t, 0),(2 t+2, t+1,1) & 2,(0,1,1) \\
(2 t+1, t, 0),(t+1,2 t+2,2) & 2,(2,1,0) \\
(2 t+1, t, 0),(1, t, 0) & 2,(1,1,2) \\
\left(2 t^{2}+1,2 t, t^{2}\right),\left(2, t, 2 t^{2}\right) & 2,(t, 0,0) \\
\left(t^{2}+t+1, t^{2}+2 t, t^{2}\right),\left(2 t+2,2 t^{2}+t, 2 t^{2}\right) & 1,(t, 0,0) \\
\left(t^{2}+2 t+1,2 t^{2}+2 t, t^{2}\right),\left(t+2, t^{2}+t, 2 t^{2}\right) & 1,(t, 0,0)
\end{array}
$$


6. Computational aspects

In our calculations we used KANT [1]. All computations took just some seconds.

\section{REFERENCES}

[1] DABERKOW, M.- FIEKER, C.,- KLÜNERS, J.,- POHST, M.ROEGNER, K. - WILDANGER, K.: KANT V4, J. Symbolic Comput. 24(1997), 267-283. http://www.math.tu-berlin.de/ Kant/

[2] GAÁL, I.: Diophantine equations and power integral bases, Boston, Birkhäuser, 2002.

[3] GAÁL, I.- POHST, M.: Diophantine equations over global function fields I: The Thue equation, J. Number Theory 119, (2006), 49-65.

[4] GAÁL, I.- POHST, M.: Diophantine equations over global function fields II: S-integral solutions of Thue equations, Experimental Math. 15, (2006), $1-6$.

[5] GAÁL, I.- POHST, M.: Diophantine equations over global function fields $I V$ : S-unit equations in several variables with an application to norm form equations, J. Number Theory 130, (2010), 493-506.

[6] LANG, S.: Algebra, Graduate Texts in Mathematics, 211, Springer, 2002.

[7] SHOREY, T.N.- TIJDEMAN, R.: Exponential Diophantine equations, Cambridge Tracts in Mathematics 87, Cambridge University Press, 2009.

[8] DE WEGER, B.M.M.: The weighted sum of two S-units being a square, Indag. Math., New Ser. 1, (1990), 243-262.

\footnotetext{
* University of Debrecen, Mathematical Institute H-4010 Debrecen Pf.12.

** Technische Universtät Berlin, Institut Für Mathematik Strasse Des 17. Juni 136, Berlin GERMANY

E-mail address: pohst@math.tu-berlin.de
} 